\title{
Lifetimes of Hypersonic Phonons in Solid ${ }^{4} \mathrm{He}^{*}$
}

\author{
P. Leiderer, P. Berberich, S. Hunklinger, and K. Dransfeld \\ Phesik Deparment der Technschen Unversiäl Mänchen \\ Munch. (jermany
}

\section{Introduction}

The quantum liquid "superfluid helium" shows several outstanding properties as compared to normal liquids. In contrast to this the quantum crystal "solid helium" (in the following we shall only consider ${ }^{4} \mathrm{He}$ ) seems to exhibit no remarkable differences with respect to ordinary solids. For example, solid helium was found to have well-defined crystal structures, ${ }^{1}$ specific heat and sound velocity measurements remained without surprise, ${ }^{2.3}$ and neutron scattering experiments led to the usual dispersion spectrum for phonons. ${ }^{4 \cdot 6}$ After initial difficulties due to the large zeropoint motion the theoretical treatment of these phonons has been quite successful. Using techniques like self-consistent field methods, the calculations for the phonon frequencies are in fair agreement with all these experiments. ${ }^{7}$

If one considers the attenuation of the phonons, the problem is not yet settled. Because of the large zero-point energy the atoms move far beyond the harmonic range of the interatomic potential. Therefore, at first glance, one might expect such a crystal to be highly anharmonic and, as a consequence, to show considerably higher damping of lattice waves than classical crystals. Jäckle and Kehr, ${ }^{8}$ however, showed that the classical theory of ultrasonic absorption should also be applicable to anharmonic crystals if the actual interatomic potential is replaced by a renormalized one. The important parameters entering into such a calculation are the higher-order elastic constants of the crystal, which roughly are represented by the Grüneisen constant. The value of about 3 of the Grüneisen constant in solid helium $^{5.9 .10}$ is quite close to that of heavier rare gas solids and of many other dielectrics.

What is to be expected for the attenuation of sound at low temperatures $(T \ll \theta)$ from the classical theory? Under the basic assumption that the attenuation of ultrasonic phonons in an ideal crystal is caused by their interaction with thermal phonons, the following expression for the inverse lifetime (in $\sec ^{-1}$ ) of longitudinal ultrasonic phonons has been derived ${ }^{11}$ :

$$
\tau^{-1}=\frac{\hbar \gamma^{2}}{32 \pi^{2} \rho l_{i}^{5}}\left(\frac{4 \pi^{4}}{15}\right)\left(\frac{k_{\mathrm{B}} T}{\hbar}\right)^{4} \omega \tan ^{-1}\left(\omega \tau_{\mathrm{th}}\right)
$$

where $y$ is an effective Gruneisen constant, $\omega$ and $v_{1}$ are the ultrasonic angular frequency and longitudinal velocity, respectively, and $\tau_{\mathrm{th}}$ is the lifetime of longitudinal thermal phonons.

* Supported by the Deutsche Forschungsgemcinschaft. 
For $\omega \tau_{\text {th }} \ll 1$ Eq. (1) leads to

$$
\tau^{-1} \propto \omega^{2}
$$

The temperature dependences of $\tau_{\mathrm{th}}$ and of the prefactor nearly compensate in this regime.

At low temperatures the mean free path of the thermal phonons becomes much longer than the wavelength of the ultrasonic phonons, i.e., $\omega \tau_{\mathrm{th}} \gg 1$. Then the arctan is near $\pi / 2$, resulting in

$$
\tau^{-1} \propto \omega T^{4}
$$

This is known as the Landau-Rumer relation.

Our experiment was conducted in the temperature range from 1 to $2^{\circ} \mathrm{K}$. It is known from measurements of the thermal conductivity that the mean lifetime of thermal phonons in this region is limited both by normal and Umklapp processes and is

$$
\left\langle\tau_{\mathrm{th}}\right\rangle>10^{-9} \mathrm{sec}
$$

The frequency of sound in our experiment is

$$
v \geqq 1.5 \mathrm{GHz}
$$

Assuming Eq. (4) also to be valid for the longitudinal thermal phonons, we get

$$
\omega \tau_{\mathrm{Lh}} \gg 1
$$

and we should therefore expect relation Eq. (3) to apply.*

This relation is well satisfied for many classical dielectric crystals. In order to compare different substances, reduced coordinates $T / \theta$ and $\omega / \omega_{\mathrm{D}}$ have been used, where $\theta$ and $\omega_{\mathrm{D}}$ are Debye temperature and Debye frequency, respectively. Then from Eq. (1) we obtain

$$
\tau^{-1} \propto \gamma^{2}\left(\frac{\rho^{2}}{m^{5}}\right)^{1 / 3}\left(\frac{\omega}{\omega_{\mathrm{D}}}\right)\left(\frac{T}{\theta}\right)^{4} \tan ^{-1}\left(\omega \tau_{\mathrm{th}}\right)
$$

Some experimental results are shown in Fig. 1. We have plotted the inverse lifetimes of the hypersonic phonons multiplied by a normalizing factor due to Eq. (5), versus the reduced temperature. In our temperature regime, for $T / \theta<0.075$, the inverse lifetime has the same temperature dependence in $\mathrm{KCl}_{,}{ }^{14} \mathrm{NaCl}^{15}{ }^{15} \mathrm{SiO}_{2},{ }^{16}$ and $\mathrm{Al}_{2} \mathrm{O}_{3},{ }^{17}$ namely

$$
\tau^{-1} \propto T^{4}
$$

The difference in the absolute value is only due to the different Grüneisen parameters, which for the quoted substances vary from 1 to 3 . The dashed line in Fig. 1 shows the behavior expected for solid helium from this classical theory.

At this point some reservation has to be made: Assuming a pressure of the solid

* Equation (1) docs nol include effects arising from the curvature of the phonon dispersion curve. Therefore it is only valid if $\omega \tau_{t h}<2(\theta i T)^{2}$, which in solid helium is fulfilled for our experimental frequencies if $T>1{ }^{\circ} \mathrm{K} .{ }^{23}$ 
Fig, 1. Reduced inverse lifetime of hypersonic phonons in diclectric crystals. The data for $\mathrm{KCl}, \mathrm{NaCl} . \mathrm{SiO}_{2}$, and $\mathrm{Al}_{2} \mathrm{O}_{3}$ are taken from experiment. The dashed line indicates the expected behavior of solid helium. Circles and squares represent the experimental data of solid helium.

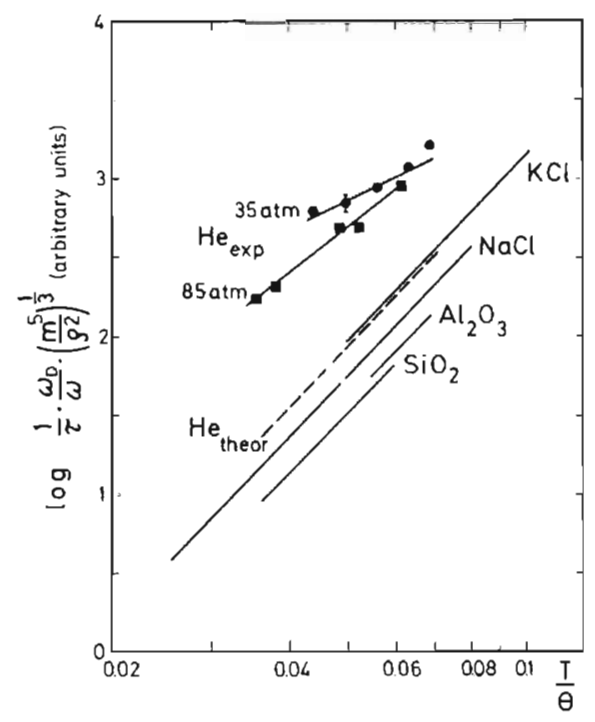

helium sample of $35 \mathrm{~atm}$, as in most of our experiments, the crystal already melts at $2^{\circ} \mathrm{K}$, whereas the Debye temperature is $28^{\circ} \mathrm{K}$. So the reduced melting temperature is $T_{M} / \theta=0.075$, more than a factor of ten lower than for other substances. Very near the melting temperature a deviation from the classical behavior would not be surprising. Slightly below $T_{M} / \theta$, however, all the thermodynamic properties of solid helium can be reasonably well approximated in terms of the low-temperature limits. From this consideration one should also expect the Landau-Rumer theory to be applicable in this regime.

Until now experiments on the attenuation of phonons in solid helium which can be compared with this theoretical prediction are quite rare. From neutron scattering experiments it is known that well-defined phonons exist up to the highest frequencies in the dispersion spectrum, although some branches show an anomalous broadening. For ultrasonic frequencies near $10 \mathrm{MHz}$ measurements by Vignos and Fairbank, ${ }^{3}$ Lipschultz and Lec, ${ }^{18}$ and Crepeau et al. ${ }^{19}$ resulted in attenuation values of $0.2-0.7 \mathrm{~cm}^{-1}$, which is slightly higher than in liquid helium near $1^{\circ} \mathrm{K}$, both for longitudinal and transverse sound waves in hcp as well as in bcc ${ }^{4} \mathrm{He}$.

As the authors themselves indicate, however, until now such conventional ultrasonic measurements using transducers only give an upper limit for the intrinsic absorption in solid helium, since they are subject to large parasitic attenuation effects. Because of the pronounced acoustical anisotropy of solid helium, sound waves generally do not propagate perpendicular to the transducer surface and are therefore partially absorbed at the chamber walls. ${ }^{20}$ Furthermore, ultrasonic measurements require a high degree of crystal perfection over the whole sample volume. Finally, the mechanical contact between the transducer and the sample must not vary with temperature.

All these difficulties can be avoided or become unimportant if instead of using transducers the ultrasonic phonons are generated and detected by means of light scattering. With such a technique we could measure for the first time the intrinsic 
attenuation of ultrasonic phonons in solid ${ }^{4} \mathrm{He}$ and also determine the velocity of these phonons in the $\mathrm{GHz}$ range.

\section{Experimental}

Generation of the phonon field was achieved by means of stimulated Brillouin scattering using a giant pulse ruby laser. ${ }^{21}$ The wavelength of these phonons is nearly constant and given by

$$
\lambda_{S}=i_{I} / 2 n
$$

where $\lambda_{L}$ is the wavelength of the laser light and $n$ is the refractive index of the sample.

Due to the strongly pressure-dependent sound velocity, this corresponds to phonon frequencies of 1.5 and $2 \mathrm{GHz}$ for helium crystals at 35 and 85 atm, respectively. The velocity can be determined from the frequency shift of the backscattered Brillouin light with respect to the laser light, which is equal to the phonon frequency $v_{s}$ :

$$
v_{L}-v_{B}=v_{S}=2 n v_{S} / \lambda_{L}
$$

In order to determine the absorption we employed a method which is a further improvement of a technique first used by Winterling and Heinicke. ${ }^{22}$

The setup is schematically shown in Fig. 2. Light from a second ruby laser which is weak enough not to disturb the phonon population is directed onto the hypersonic wave generated by the giant pulse. This probing light is partly backscattered by the hypersonic phonons. The backscattered intensity dies away as the phonons decay. Thus from the time-dependent ratio of backscattered to incident light the phonon lifetime can be obtained.

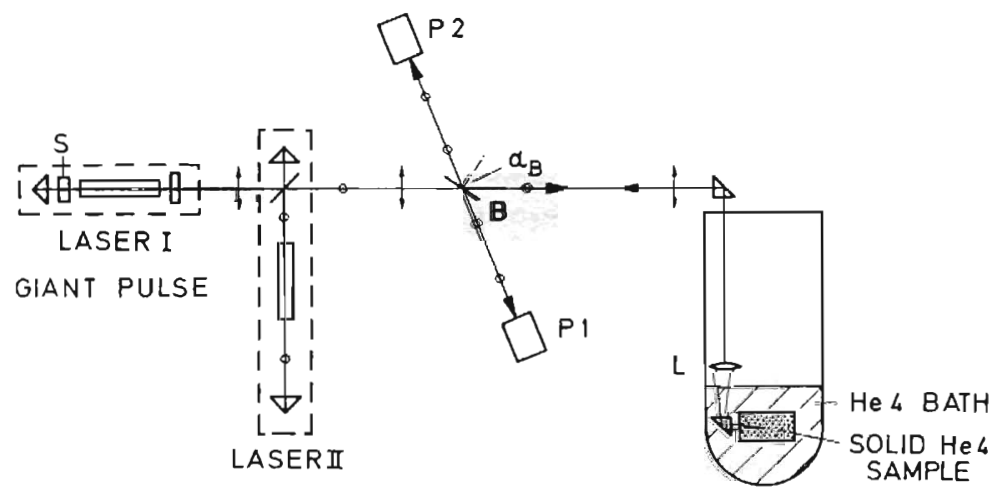

Fig. 2. Experimental setup for measuring phonon lifetimes in solid helium. Polarization of laser I (indicated by arrows) is in the plane of the page; polarization of laser II is perpendicular to it (indicated by circles). The laser light is focused into the sample chamber with lens $L(f=20 \mathrm{~cm})$. Photocells $P I$ and $P 2$ record incident and backscattered light respectively. Registration of the giant pulse light is strongly suppressed because the beam splitter $B$ is mounted at the Brewster angle $\alpha$. The electrooptical shutter $S$ is used for synchronization of the two lasers. 
Fig. 3. Upper: Typical incident light intensity registered by photocell PI of Fig. 2. The giant pulsc is suppressed by a factor of 500 . The long, regularly spiked train of pulses is duc to the second laser. Lower: Backscattered light intensity registered by photoccil P2.
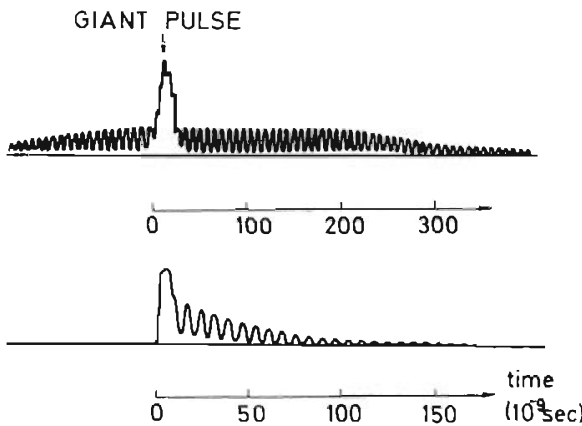

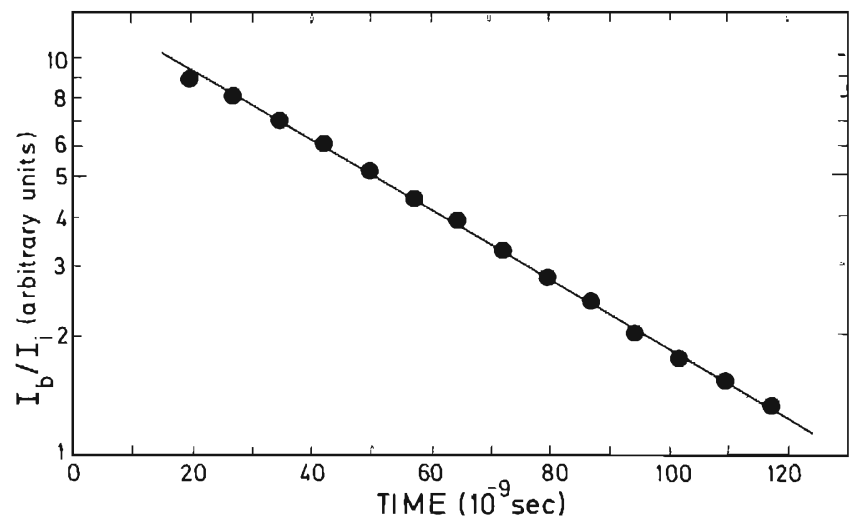

Fig. 4. Ratio of backscattered to incident light $I_{b} / I_{i}$ plotted for intensities of Fig. 3.

The upper half of Fig. 3 shows a typical oscilloscope trace of the incident light; the lower curve represents the backscattered light intensity. The ratio of backscattered to incident light is plotted in Fig. 4 as a function of time. The straight line indicates an exponential decrease of the number of phonons and yields a wclldefined lifetime, which is $50 \mathrm{nsec}$ in this case. In this way the absolute value of the lifetime may be obtained from the record of a single laser pulse.

The advantages of this method are evident: (a) No transducer is needed, so coupling problems are eliminated. (b) The crystal has to be of high quality only in the small volume where the laser light is focused. (c) Because of the short distance through which the phonons travel during measurement $(<200 \mu \mathrm{m})$ "walk off" effects are neglible.

Nevertheless, as the method described above is new, we tested it with liquid helium, where the attenuation near $1 \mathrm{GHz}$ is known from a number of experiments. We obtained exactly the same results as, for instance, St. Peters et al. ${ }^{23}$ who used a spontaneous Brillouin scattering technique. 


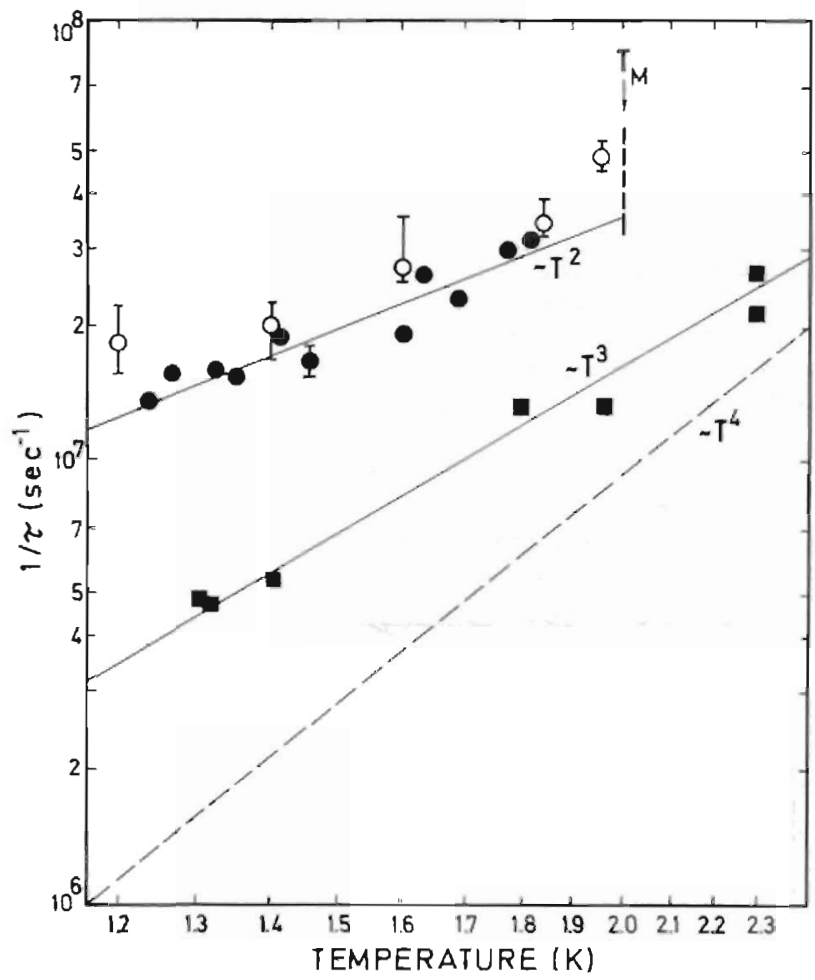

Fig. 5. Inverse lifetime of longitudinal phonons in solid ${ }^{4} \mathrm{He}$. Open and full circles represent two different hcp crystals at $35 \mathrm{~atm}$; $T_{M}$ denotes the melting temperature of these crystals. The squares are data from an $85-\mathrm{atm}$ hcp crystal. The wavelength of the phonons is $3400 \AA$, which corresponds to phonon frequencies of 1.55 and $2.02 \mathrm{GHz}$ for 35 and $85 \mathrm{~atm}$, respectively. The dashed line indicates the expected $T^{4}$ temperature dependence.

\section{Results}

As to the velocity of $1.5-\mathrm{GHz}$ longitudinal phonons in solid helium, our results were found to be in agreement with the previous data of Vignos and Fairbank, ${ }^{3}$ Crepeau et al., ${ }^{19}$ and several other groups, obtained by ultrasonic techniques at lower frequencies.

Regarding the attenuation of these phonons, however, the results were unexpected. Some data are shown in Fig. 5. We have plotted the inverse phonon lifetimes for three different hop crystals at pressures of 35 and $85 \mathrm{~atm}$ in the temperature range from 1.2 to $2.3^{\circ} \mathrm{K}$.

The data for the two 35-atm crystals are representative also of several other experimental runs under equal conditions. Apart from the point at $1.95^{\circ} \mathrm{K}$, very near the melting temperature of ${ }^{4} \mathrm{He}$ at $35 \mathrm{~atm}$, the inverse phonon lifetime (in $\mathrm{sec}^{-1}$ ) of these crystals approximately follows the relation

$$
\tau^{-1}=10^{7} T^{P}, \quad 1.5 \leqq P \leqq 2 ; \quad 1.2 \leqq T \leqq 1.95^{\circ} \mathrm{K}
$$

at a phonon frequency of $1.5 \mathrm{GHz}$ 
The data for the 85 -atm crystal are quite recent and somewhat preliminary. They can be represented by

$$
\tau^{-1}=2 \times 10^{6} T^{3} \quad 1.3 \leqq T \leqq 2.3^{\circ} \mathrm{K}
$$

\section{Discussion}

Two results may be derived from Fig. 5: In the first place, the observed temperature dependence is weaker than the expected $T^{4}$ law, the disagreement being higher for the low-pressure crystals. In this context it is interesting to note that also for thermal phonons the relaxation time for normal processes shows a weaker temperature dependence than one would expect classically: Poiseuille flow and second-sound data ${ }^{12.24}$ lead to a $\tau_{N}^{-1} \propto T^{3}$ dependence between 0.3 and $0.8^{\circ} \mathrm{K}$ compared to the relationship $\tau_{N}^{-1} \propto T^{5}$ expected classically. ${ }^{25}$

Second, the lifetimes of the phonons increase as the pressure is raised. According to the classical theory [see Eq. (1)], this increase should vary as $v_{l}^{4}$ if the wave vector is held constant as in our experiment, with a small correction due to the change in density. The mean longitudinal velocity changes from $520 \mathrm{~m} / \mathrm{sec}$ at $35 \mathrm{~atm}$ to $680 \mathrm{~m} / \mathrm{sec}$ at $85 \mathrm{~atm}$, leading to an expected increase of $\tau$ by a factor of three, which is in fair accordance with our observation. Since the orientation of the crystal has not been determined so far and the attenuation is supposed to be anisotropic, this agreement may be somewhat fortuitous.

For a comparison with classical crystals we have plotted our data also in Fig. 1. Here a further discrepancy becomes obvious: The experimental reduced lifetimes in solid helium are much shorter than expected, more than one order of magnitude at the lowest temperatures. (This also holds for bcc ${ }^{4} \mathrm{He}$, where the lifetimes turned out to be comparable to that in the hcp phase in the immediate neighborhood.) Again the discrepancy is somewhat smaller for the higher-pressure crystal.

In order to explain these discrepancies, one could either assume that they are due to an improper choice of the parameters entering into the calculations, or that an additional damping mechanism comes into play in solid helium.

Let us first consider how the parameters had to be changed in order to obtain agreement with classical theory.

(a) The high absolute value of the inverse phonon lifetime would require the anharmonic parameter $\gamma$ of Eq. (1) to have a value exceeding ten at $1.2^{\circ} \mathrm{K}$. This is more than a factor of three larger than the Grüneisen constant as determined from specific heat, ${ }^{9}$ sound velocity, ${ }^{10}$ and neutron scattering experiments. ${ }^{5}$

(b) The weak temperature dependence of $\tau$ could be explained if the condition $\omega \tau_{1 k} \gg 1$ which is necessary for a Landau-Rumer behavior were not fulfilled, but only $\omega \tau_{\mathrm{th}} \geqslant 1$. This would lead to relaxation times of the order $\tau_{\mathrm{th}} \sim 10^{-10} \mathrm{sec}$, which is more than two orders of magnitude smaller than the observed mean thermal value $\left\langle\tau_{\text {:h }}\right\rangle$ at $1.2^{\circ} \mathrm{K}^{12}$

Therefore it seems to be more probable that some other damping mechanism contributes to the phonon attenuation in solid helium, although at the moment there exist only speculations about its origin:

(a) One possibility could be nonlinear effects, like the generation of harmonics, because of the relatively high amplitude of the sound wave in our experiment. 
Applying our technique to liquid helium and quartz, however, where the buildup time for shock wave formation should be comparable to that in solid helium, ${ }^{26}$ we did not observe such effects.

(b) Furthermore, some contribution from zero-point phonons ${ }^{27}$ could decrease the phonon lifetime, although we are lacking a model for this mechanism so far.

(c) One might also think of an interaction between acoustic Brillouin phonons and some other excitations present in solid helium. For instance, regarding the similarity of the excitation spectra of liquid and solid helium, ${ }^{28}$ one could assume the damping to be due to a relaxation process between acoustic and optical phonons similar to the phonon-roton relaxation in liquid helium. This idea is supported by the pronounced similarity we found for the attenuation curves in liquid helium near $24 \mathrm{~atm}$ and in the solid near $30 \mathrm{~atm}$. In order to clear up these questions, experiments at lower temperatures and various frequencies are in progress.

\section{Summary}

Using a light scattering technique, we have determined the velocity and the lifetime of longitudinal $\mathrm{GHz}$ phonons in solid ${ }^{4} \mathrm{He}$. Comparing our results for the phonon lifetime with the classical theory, we have found a much smaller absolute value and a weaker temperature dependence than expected. This disagreement seems to decrease for crystals at higher pressure.

\section{Acknowledgment}

We are grateful to Professor R. Nava for helplul discussions.

\section{References}

1. J. Wilks, Properties of Liquid and Solid Helium, Clarendon Press, Oxford (1967), p. 589

2. D.(). Edwards and R.C. Pandorf, Phrs. Rev, 140, A816 (1965); 144, 143 (1966).

3. J.H. Vignos and H.A. Fairbank, Phys. Rev. 147, 185 (1966).

4. V.J. Minkiewicz, T.A. Kitchens, F.P. Lipschultz, R. Nathans, and G. Shirane, Phys. Rev. 174, $267(1968)$

5. R.A. Recse, S.K. Sinha, T.O. Brun, and C.R. Tilford, Phys, Rev. A 3, 1688 (1971)

6. E.B. Osgood, V.J. Minkiewicz, T.A. Kitchens, and G. Shirane, Phys. Rev. A 5, 1537 (1972).

7. N.R. Werthamer, Am. J. Phys. 37, 763 (1969).

8. J. Jäckle and K.W. Kehr, Phys. Rev. Lett. 24, 1101 (1970).

9. G. Ahlers, Phy.s. Lett. 24A, 152 (1967).

10. R. Wanner and J.P. Franck, Phys. Rev. Lett. 24, 365 (1970).

11. P.C.K. Kwok, in Solid State Physics, F. Seitz, D. Turnbull, and H. Ehrenreich, eds., Ncw York London (1967), Vol. 20, p. 213.

12. E.M. Hogan, R.A. Guyer, and H.A. Fairbank, Phys. Rev. 185, 356 (1969)

13. H.J. Maris, Phil. Mag. 9, 901 (1964); J.B. Thaxter and P.E. Tannenwald, IEEE Trans. on Sonics and Ultrasonics SU13, 61 (1966)

14. R.P. Auyang, Thesis, Cornell University, 1968.

15. L.G. Merkulov, R.V. Kovalenok, and E.V. Konovodchenko, Soviet Phys.-Solid State 13.968 (1971).

16. M.F. Lewis and E. Patterson, Phys. Rev. 159, 703 (1967).

17. J. de Klerk, Phys. Rev. 139, Al635 (1965).

18. F.P. Lipschultz and D.M. Lee, Phys. Rev. Lett. 14, 1017 (1965).

19. R.H. Crepeau, O. Heybey, D.M. Lee, and S.A. Strauss, Phys. Rev. A 3. 1162 (1971).

20. R. Wanner, Phys. Rev. A 3, 448 (1971). 
21 R.J. Chiao, C.A. Townes, and B.P. Stoicheff, Phys. Rev. Lett. 12, 592 (1964).

22. (j. Winterling and W. Heinicke, Phys. Lett. 27A, 329 (1968).

23. R.L.St. Peters, T.J. Greytak, and G.B. Benedek, Optics Commun. 1, 412 (1970).

24. C.C. Ackerman and R.A. Guyer, Ann. Phys. (N.Y.) 50, 128 (1968)

25. H.E. Jackson and C.T. Walker, Phys, Rev. B 3, 1428 (1971).

26. R.T. Beyer and S.V. Letcher, Physical Ultrasonics, Academic Press, New York (1969), p. 202.

27. G. Meissner, private communication.

28. O.W. Dietrich, E.H. Graf, C.H. Huang, and L. Passel, Phys. Rev. A 5, 1377 (1972). 